\title{
Optical Trap Combined with Raman Spectroscopy to Probe Red Blood Cell Behaviour in Certain Intravenous Fluids
}

\author{
Jijo Lukose ${ }^{1}$, Ganesh Mohan ${ }^{2}$, Mithun $\mathbf{N}^{1}$, Shamee Shastry ${ }^{2}$, and Santhosh $\mathbf{C}^{{ }^{*}}$ \\ ${ }^{1}$ Centre of Excellence for Biophotonics, Department of Atomic and Molecular Physics, Manipal Academy of Higher \\ Education, Manipal, Karnataka 576104, India \\ ${ }^{2}$ Department of Immunohematology and Blood Transfusion, Kasturba Medical College, Manipal Academy of Higher \\ Education, Manipal, Karnataka 576104, India \\ *e-mail: santhosh.cls@manipal.edu
}

\begin{abstract}
Systematic studies on a live, individual human red blood cell without any chemical fixation can be realized with the aid of optical tweezers. In the present work, the behavior of human red blood cell in dextrose containing intravenous fluids and blood plasma were investigated and compared using a home-made near-IR Laser Tweezers Raman spectrometer. Significant spectral variations in Raman signatures exist between the RBCs in plasma and dextrose containing intravenous fluids, which mainly comprise of the hemoglobin oxygenation-deoxygenation transition as well as the heme aggregation markers. (C) 2019 Journal of Biomedical Photonics \& Engineering.
\end{abstract}

Keywords: Raman spectroscopy; Optical Tweezers; Red Blood Cells; Intravenous fluids.

Paper \#3338 received 6 Nov 2019; revised manuscript received 12 Dec 2019; accepted for publication 20 Dec 2019; published online 31 Dec 2019. doi: 10.18287/JBPE19.05.040302.

\section{Introduction}

Cellular investigations are of high interest in biomedical scientific fraternity for the monitoring of cell-drug interaction, cellular abnormalities, cells under stress, cell adhesion, single cell transfection, etc. [1]. The global drive towards cytometry research has generated keen interest amongst biophotonics community to make it a major focus of interest with the aid of various optical tools. In general, cytometric studies are highly dependent upon exogenous fluorescent dyes for site specific labeling of biomolecules to acquire biochemical details in the cell. Despite its ubiquitous use, fluorescence labeling technique suffers major pitfalls, which includes extensive sample preparation, time consumption, and photo bleaching, etc. [2]. Raman spectroscopy is a widely established spectroscopic approach used for extracting pivotal information from biological samples (cells, tissues, etc.) in absence of any exogenous labelling agents [1, 3-5]. This nondestructive technique is based on analyzing the inelastic scattering of photons from the sample activated by monochromatic light from a laser source. Raman spectra of a sample are indeed a molecular fingerprint carrying vital information regarding its biochemical composition, molecular structure and function.
Conventional Raman spectroscopy has the limitation for single cell investigation due to the random motion of live cells suspended in a liquid media. The analytical capabilities of Raman technique are thus constrained by the inability to manipulate, as well as analyze individual cells of interest in absence of any cell fixative agents. Optical Tweezers developed by Arthur Ashkin, which employs tightly focused laser beam for trapping the dielectric particles, have been a revolutionary approach in the field of single cell research [6]. Ashkin et al., have used optical trapping technique for the manipulation of various particles with dimensions ranging from $100 \mathrm{~nm}$ to 10 microns that includes mammalian cells, bacteria, spores and polymer beads [7, 8]. This technique is highly advantageous compared to the other live cell immobilization methods (physical as well as chemical fixation methods), which will affect its chemical microenvironment and may adversely affect cellular functions [9]. The combination of optical trapping along with spectroscopic techniques paves researchers a better approach for extracting biochemical information at individual cell level.

Raman Tweezers can be thus very promising for probing live individual cells in absence of any exogenous labels and experiments can be also performed in aqueous solutions since water is a weak 
Raman scatterer. Li et al. have used this technique for the sorting and identification of live and dead yeast cells in a batch culture, which has showed its ability to use in microbiology for the rapid detection of food-borne pathogens [10]. Recently our group has also demonstrated the ability of Raman Tweezers combined with multivariate data analysis technique for diagnosing and classification of bacteria responsible for urinary tract infections [11]. Last decade has been witnessing considerable advancements in micro-Raman combined optical tweezers for the characterization of red blood cells for numerous biomedical applications. This includes the detection of various blood disorders as well as evaluating the stress induced by different external factors such as mechanical stretching, chemicals, etc. [12-15]. The ability of Raman Tweezers for investigating blood disorders such as alpha- and betathalassemia was also reported, primarily based on evaluating the hemoglobin oxygenation capability of red blood cells [16]. In a similar fashion, present technique was also employed for studying malarial infected blood cells and observed a reduced hemoglobin-oxygen affinity as compared to normal blood cells [17]. Petrov et al. have reported the hemoglobin oxygenation to deoxygenation switching in case of mechanically stretched red blood cells [18]. Chan et al. observed that red blood cells from sickle cell anemia patients were highly susceptible to deoxygenation under mechanical force with respect to red blood cells from healthy volunteers [12]. Feng et al. have investigated the feasibility of Raman tweezers in combination with multivariate statistical tool (PCA-LDA), for the discrimination of Type $\mathrm{AB}$ from other blood group, with an accuracy of $100 \%$ [19]. Similarly, researchers were able to detect type II diabetes with a sensitivity of $100 \%$ by combining multivariate analysis with Raman spectra [20].

Even though, the application of Raman Tweezers in transfusion medicine is highly limited. Administration of intravenous infusions in clinical settings is a topic which demands serious attention in perspective of maintaining integrity of blood cells and its functioning. Use of various intravenous fluids for critically ill patients in intensive care settings is so ubiquitous in the daily hospital settings. As per the "NICE 2015" report, one among the five patients administrated with IV fluids and electrolytes in hospitals face health complications due to inappropriate infusion [21]. Even though it is in wide use, scientific evidence regarding the optimal composition and administered volume of IV fluids in clinical settings are not yet thoroughly investigated. Infusing an inappropriate IV fluid may cause undesirable complications along with less than optimal patient outcome, a major cause of patient morbidity and mortality [22]. Inappropriate fluid infusion may cause derangement of serum sodium concentration, which can in some cases even raise complicated neurological disorders. Dextrose containing intravenous fluids was utilized in clinical settings for IV fluid replacement in adult elective surgery [23]. Currently, there is a lack of sufficient proof to substantiate the application of dextrose-composed fluids when initiating intravenous fluid replacement in the adult elective surgical population [23]. Proper fluid management as well as better understanding of IV fluids plays very vital role in the treatment of all critical patients. The present work involves the comparative study of the behavior of live red blood cells in dextrose containing intravenous fluids and blood plasma probed using Laser Raman Tweezers. Micro-Raman spectroscopy can be effectively used for probing the influence of chemical environment on a sample of interest, since molecular vibrations of the sample relies on its molecular conformation as well as its external environment. The study has high implications in understanding the response of human live cells towards the stress effects in dextrose composed intravenous fluids. To the best of our knowledge, spectroscopic investigation of dextrose based IV solutions interaction with red blood cells is not yet reported. This is the first report from the Raman perspective on the spectral variations occurring to live human red blood cells under the influence of clinically used Dextrose normal saline (DNS) and 5\% Dextrose (5D) in water.

\section{Methods}

Ethical approval was obtained from the Institutional Ethics Committee, Kasturba Medical College and Kasturba Hospital, (IEC: 68/2018) for the present study. Both Dextrose Normal Saline and 5\% Dextrose in water were procured from Otsuka suppliers. The present work was performed using a custom built single beam Raman Tweezers set up (Fig. 1). Blood obtained from healthy subjects after informed consent was highly diluted (1 $\mu 1 \mathrm{RBC}$ : $5 \mathrm{ml}$ solvent) using IV fluids solution in order to avoid multiple cells. Both trapping as well as Raman characterization were carried out using the $785 \mathrm{~nm}$ laser source (Starbright Diode Laser, Torsana Laser Tech, Denmark) (Fig. 1). Holographic Band pass filter was used after the laser in order to block the extraneous $790 \mathrm{~nm}$ line from the laser source. Beam expander (BE) was used for increasing the beam diameter (nearly $8 \mathrm{~mm}$ ) so as to overfill the back aperture of the microscope aperture after which, beam was directed into an inverted microscope (Nikon Eclipse, Ti-U, Japan). A dichroic mirror (DM) was used to reflect the $785 \mathrm{~nm}$ into a $100 \mathrm{x}$ oil immersion microscope objective (MO). The beam was focused into the single cell for trapping. Backscattered light from the trapped particle or cell was directed into spectrometer (Horiba Jobin Yvon iHR320 with 1200 grooves $/ \mathrm{mm}$ grating blazed at $750 \mathrm{~nm}$ ) via same microscope objective. Raman signal was detected via liquid nitrogen cooled CCD (Symphony CCD-1024x256-OPEN-1LS). Initial calibration of the system was performed using commercially procured polystyrene beads. Visualization of the blood cells suspended in IV fluids was acquired using the camera fixed at one of the exit ports of the microscope. Baseline correction of the recorded spectra was done by means of asymmetric least squares 
smoothing method in MATLAB. Vector normalization method was used for normalizing the Raman spectra. Raman spectrum of the trapped cell was recorded at an exposure time of 60 seconds with a laser power of $\sim 7 \mathrm{~mW}$. Raman spectra of $15 \mathrm{RBCs}$ each were recorded from plasma, 5D and DNS.

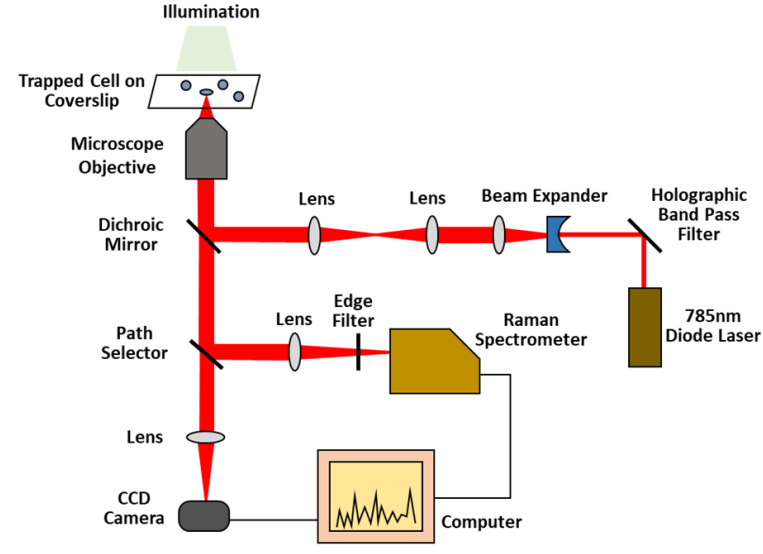

Fig. 1 Schematic of Raman Tweezers set up.

\section{Results}

The behavior of human live RBCs in dextrose containing intravenous fluids (5D and DNS) relative to blood plasma is studied using the combination of optical tweezers and Micro-Raman spectroscopy. Red blood cells suspended in $\mathrm{AB}$ ( $\mathrm{AB}+$ blood group) blood plasma were selected as the control instead of commonly used PBS buffer solution in order to mimic the real body environment (cell environment) to some extent. AB blood plasma is used in the experiments since it is regarded as a universal plasma donor. All the experiments were performed under same experimental conditions (laser power, exposure time and accumulations). Raman spectra were recorded from 15 red blood cells from a healthy subject in both IV fluids and the average of these 15 spectra are plotted in Fig. 2-4. Most of the peaks present in the Raman spectra of a trapped red blood cell are originating from the iron containing protein i.e. hemoglobin. Hemoglobin protein present inside the red blood cell is responsible for the transport of oxygen. Cells present in 5D solution displayed noticeable differences for some prominent peaks $\left(565 \mathrm{~cm}^{-1}, 972 \mathrm{~cm}^{-1}, 1209 \mathrm{~cm}^{-1}, 1222 \mathrm{~cm}^{-1}\right.$, $1368 \mathrm{~cm}^{-1}, 1636 \mathrm{~cm}^{-1}$ ) in their Raman spectra relative to the control cells, which are shown by arrows in Fig. 2 . Raman Tweezers are commonly used to investigate the oxygenation-deoxygenation transitions of intracellular hemoglobin in single red blood cell under stress conditions as well as in various abnormalities, since Raman signatures of RBCs are highly sensitive to the oxygenation state of the cell. The iron lying at the center of the porphyrin ring is responsible for the hemoglobinoxygen binding occurring in the red blood cells (Fig. 5). The iron atom lies in the porphyrin plane, acquires a planar configuration once the oxygen is bound to the sixth coordination site of iron [24]. The iron atom will be pushed apart from the porphyrin plane towards the histidine residue in case of deoxygenation, which leads to the formation of domed configuration [25]. This conformational switching during oxy-deoxy transition will be instantly reflected in the vibrational modes associated with the porphyrin ring, which can be monitored from the Raman spectra.

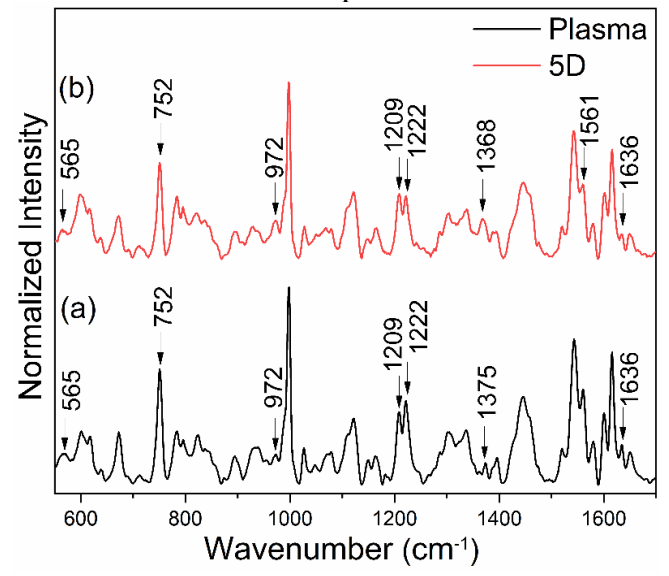

Fig. 2 Raman spectra of red blood cells suspended in (a) Blood plasma and (b) 5\% Dextrose (5D).

The intracellular oxygenated hemoglobin status is usually examined by inspecting the two major bands associated with the $\mathrm{C}-\mathrm{H}$ in-plane vibrations of methine hydrogen. In general, the band at $1209 \mathrm{~cm}^{-1}$ is considered as the prominent marker to indicate the deoxygenated hemoglobin status and $1222 \mathrm{~cm}^{-1}$ is considered as oxyhemoglobin marker [26, 15]. The conformational modifications arise in hemoglobin is capable to make instant impact in the deformation angle of $\mathrm{C}-\mathrm{H}$ vibrations. The highly sensitive response of these vibrations to the alterations in heme conformation is the result of its close proximity to protein subunits [24]. As evident from the Fig. 3(a), the $1222 \mathrm{~cm}^{-1}$ oxygenation marker is prominent for RBCs in blood plasma. Intensity switching of these major bands was observed for 5D treated RBCs, where deoxygenated hemoglobin marker at $1209 \mathrm{~cm}^{-1}$ is slightly prominent than its oxy marker. This region indicates that the oxygen binding capability of intracellular hemoglobin is affected in case of cells present in 5\% Dextrose. Spin marker region is also of major interest in case of Raman investigations on red blood cells since this region is also accompanying some major oxy-deoxy markers. The Raman markers appearing at $1561 \mathrm{~cm}^{-1}$ and $1636 \mathrm{~cm}^{-1}$ indicating the spin states of the iron atom are originated from the $\mathrm{C}-\mathrm{C}$ bonds in the porphyrin ring. As mentioned earlier, the switching of iron from the low spin ferric state (oxygenated) to high spin state results in the displacement of the $\mathrm{Fe}$ atom $4 \mathrm{~nm}$ out of the porphyrin plane. This conformational change is not only linked with the methine deformation region, but also can be tracked from the vibrational modes associated with the spin marker region [26]. This is visible from the decrease in band at $1561 \mathrm{~cm}^{-1}$ in $5 \mathrm{D}$ solution as highlighted in Fig. 2. This oxy-deoxy assumption is also well supported by the decrease in dioxygen marker band at $1636 \mathrm{~cm}^{-1}$ in the spin marker region [25]. Moreover, 

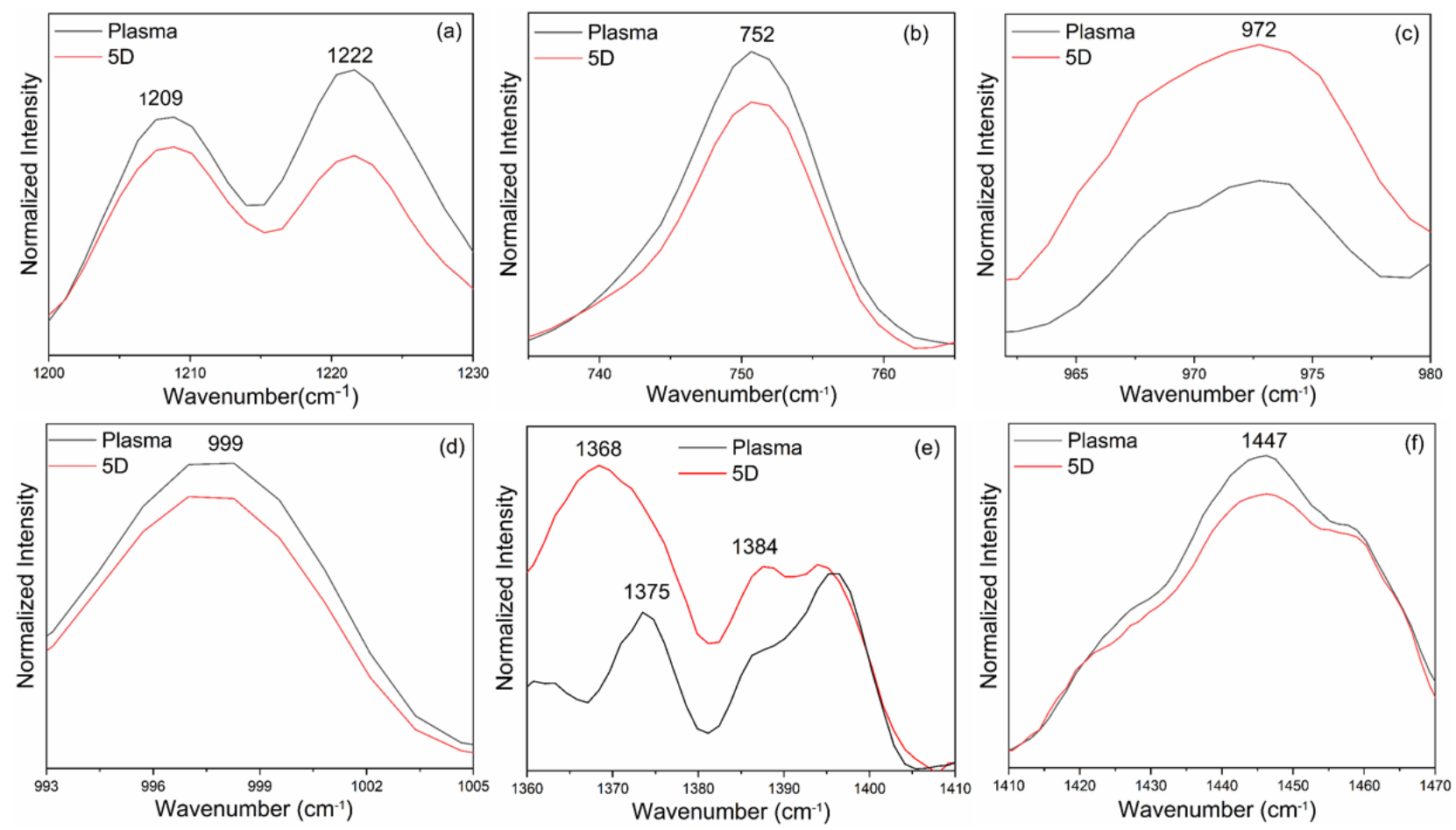

Fig. 3 Overlaid spectra of (a) Methine deformation region, (b) Porphyrin breathing mode, and (c) Heme Aggregation marker, (d) Phenyl alanine marker, (e) $\sim 1360$ to $1410 \mathrm{~cm}^{-1}$ region, and (f) $\mathrm{CH}_{2} / \mathrm{CH}_{3}$ deformation mode marker.
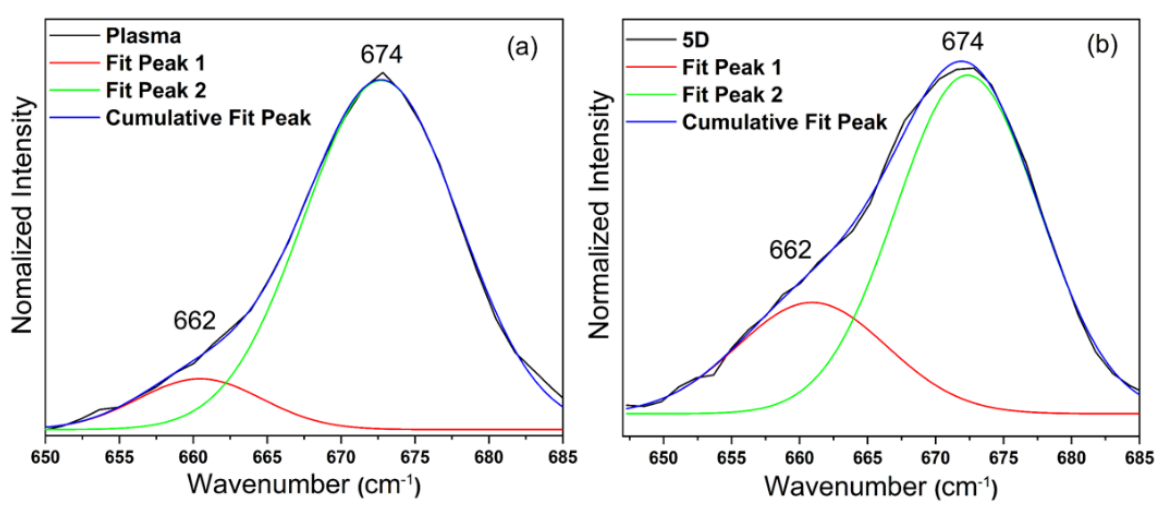

Fig. 4 Raman spectra for $\sim 650$ to $685 \mathrm{~cm}^{-1}$ region and the fitted peaks for $\mathrm{RBC}$ in (a) Blood plasma and (b) $5 \%$ Dextrose.

the markers corresponding to heme-bound oxygen $\left(\mathrm{Fe}-\mathrm{O}_{2}\right.$ stretch) present at $565 \mathrm{~cm}^{-1}$ as well as the pyrrole deformation band at $674 \mathrm{~cm}^{-1}$ experience an intensity reduction in IV fluid, which again validate the impact of IV fluid on oxygen binding affinity of heme structure in red blood cell.

The band at $752 \mathrm{~cm}^{-1}$ due to $\mathrm{C}-\mathrm{N}-\mathrm{C}$ breathing stretch in the porphyrin ring is regarded as an indicator of heme status in RBC, exhibited a lower signal in 5D solution, as compared to blood plasma (Fig. 3b) [20]. Similar trend is also obtained for the symmetric ring breathing mode of phenyl alanine present at $999 \mathrm{~cm}^{-1}$ (Fig. 3d). Since both of these vibrations are reported as sensitive to oxygen in previous literature, these intensity decreases may be due to the variation in oxidation environment in red blood cells [20,27]. On the other hand, hemoglobin depletion due to exogenous substances (e.g. alcohol) can also result in similar trend for porphyrin breathing mode [28, 29]. Apart from the above-mentioned spectral variations, which are associated with the intracellular oxyhemoglobin status, the presence of $5 \mathrm{D}$ solution resulted in the enhancement of RBC bands present at 972 (Fig. 3c), 1368 and $1384 \mathrm{~cm}^{-1}$ (Fig. 3e). An additional band at $662 \mathrm{~cm}^{-1}$ present along with the pyrrole deformation band at $674 \mathrm{~cm}^{-1}$ is enhanced in 5D solution, which is evident after performing curve resolution in this region (Fig. 4). These vibrational changes were attributed to the formation of hemoglobin aggregates resulting from protein denaturation $[27,30]$. The intensity decrease in $\mathrm{CH}_{2} / \mathrm{CH}_{3}$ deformation modes at $1447 \mathrm{~cm}^{-1}$, from amino acid side chains also hints the probable damage of 
membrane protein in presence of IV fluid as shown in Fig. 3f.

(a)

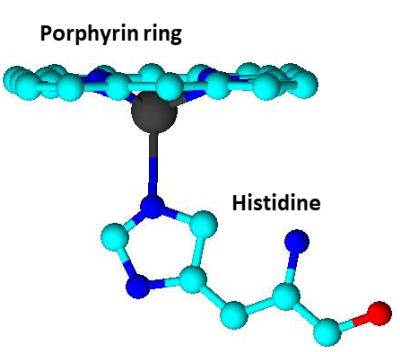

Fig. 5 Schematic of (a) deoxygenated heme and (b) oxygenated heme.

The mean Raman spectra obtained for red blood cells suspended in DNS and blood plasma are shown in Fig. 6. Similar to 5D, red blood cells in DNS also shown a deoxygenation tendency which is evident from the oxy-deoxy markers at 565, 1209, 1222, 1544, 1561 and $1636 \mathrm{~cm}^{-1}$. The major oxygenation markers, which undergone changes, are given in Fig. $7(a-c)$. At the same time, heme aggregation tendency of red cells observed in DNS solution seen from the mild enhancement in bands including 972 and $1368 \mathrm{~cm}^{-1}$ is not as significant as in $5 \mathrm{D}$. The intensity ratio of the oxy-deoxy markers presented in methine deformation region $\left(1209 \mathrm{~cm}^{-1}\right.$ and $\left.1222 \mathrm{~cm}^{-1}\right)$ in all three samples are plotted in Fig. 8(a). Similarly, the intensity ratio of oxy-deoxy markers presented in the spin marker region
(1561 $\mathrm{cm}^{-1}$ and $1544 \mathrm{~cm}^{-1}$ ) is also plotted (Fig. 8(b)). The intracellular oxygen binding ability is adversely affected in both dextrose containing IV fluids as compared to blood plasma, and it is more prominent in DNS, which is confirmed from the present microRaman studies. There may be also some contribution from laser induced deoxygenation due to optical trapping of $\mathrm{RBC}$. More studies are thus required to further elucidate this intracellular RBC oxygenationdeoxygenation transition mechanism.

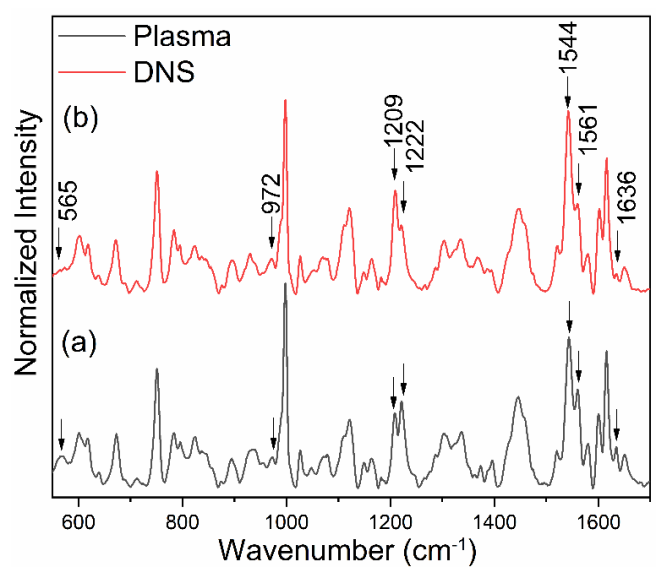

Fig. 6 Raman spectra of red blood cells suspended in (a) Blood plasma and (b) Dextrose normal saline (DNS).
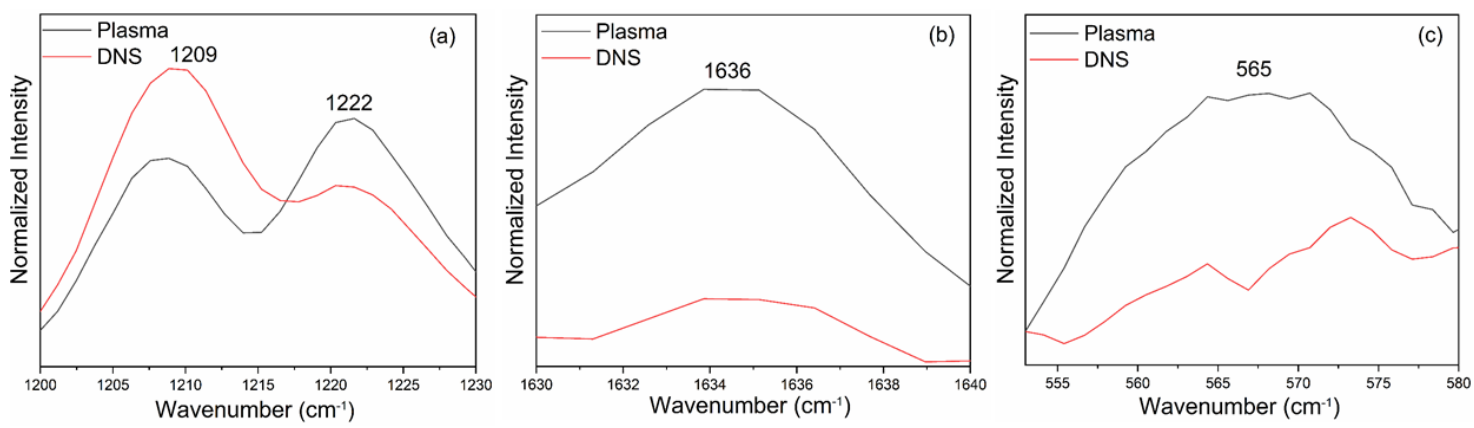

Fig. 7 Overlaid spectral plot of (a) Methine deformation region, (b) Spin marker region, (c) Fe- $\mathrm{O}_{2}$ stretch for blood plasma and Dextrose normal saline.
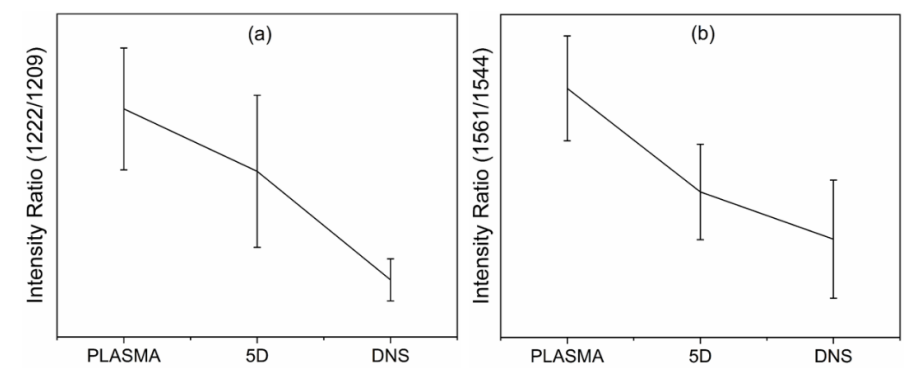

Fig. 8 (a) Ratio of relative intensities of the Raman peaks at $1222 \mathrm{~cm}^{-1}$ with respect to the $1209 \mathrm{~cm}^{-1}$ peak for RBCs suspended in different samples. (b) Ratio of relative intensities of the Raman peak at $1561 \mathrm{~cm}^{-1}$ with respect to the $1544 \mathrm{~cm}^{-1}$ peak. 


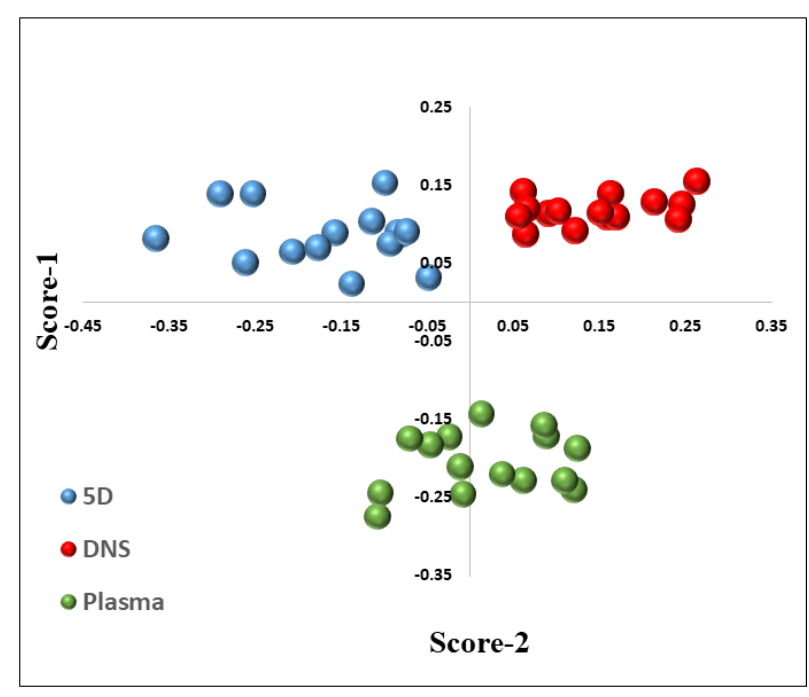

Fig. 9 PCA results obtained from the spectral analysis of control red blood cells (in plasma), red blood cells in 5\% Dextrose and Dextrose normal saline (DNS).

Principal component analysis (PCA) was also performed to acquire statistical discrimination among the Raman spectral data of blood cells dispersed in plasma and dextrose containing IV fluids (5D and DNS). This statistical analysis was performed on a total of 45 Raman spectra, in which 15 spectra each from cells diluted in plasma, 5D and DNS. Fig. 9 shows the PC1 versus PC2 scores plot for spectra from the cells suspended in blood plasma and dextrose containing IV fluids. Very good differentiation was observed among the above three classes. Quantitative Phase Images (QPI) of an individual RBC in different solutions were also recorded using d'Bioimager (d'Optron) in order to evaluate the morphological deviations from the normal discocyte shape as shown in Fig. $10(\mathrm{a}-\mathrm{c})$. Red blood cells suspended in DNS and 5D displayed significant morphological alterations in the microscopic images, which is clearly understood from quantitative phase images. Biconcave shape plays a crucial role in promoting elastic deformation of blood cell, which is a major requirement for its circulation through micro capillaries [31]. The discocyte nature of cell in plasma is evident from the phase image associated with it (Fig. 10 (a)). At the same time, sphereocytes formation were observed in hypotonic saline solution with the center portion of the RBC is bulged (Fig. 10(b)). Morphological disturbance is also observed for some cells suspended in DNS (Fig. 10 (c)). It is clear that red blood cells in Dextrose IV fluids failed to maintain its inherent biconcave shape, which indicates that cytoskeleton structure is altered. This may affect the cell deformation ability, which can hinder microcirculation through blood capillaries. Less deformability in sphereocytes makes them more vulnerable to damage during microcirculation as compared to normal red blood cells, which maintain discocyte shape.
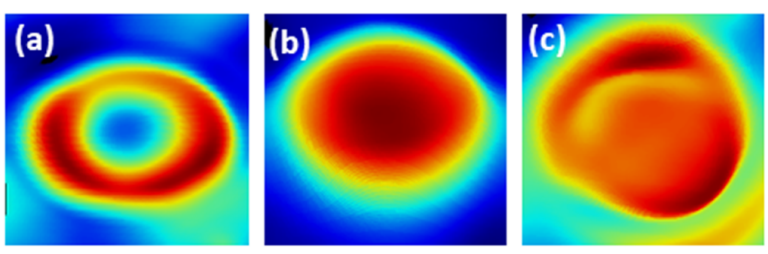

Fig. 10 Quantitative Phase images obtained for RBC in (a) Blood plasma, (b) 5\% Dextrose (5D), and (c) Dextrose normal saline (DNS).

In brief, Raman Tweezers have shown high potential in studying the exogenous agent's interaction with red blood cells. Both silver and gold nanoparticles induce oxidative stress has been studied in detail using this technique [32]. Normal Saline induced deoxygenation of red blood cells was also reported [33]. Alcohol induced stress on red blood cells was also monitored using Raman Tweezers in a recent article, where hemoglobin deoxygenation was observed. Moreover hemoglobin leakage from RBCs was identified from the decrease in porphyrin breathing mode intensity in the Raman spectra [34]. Similarly, the impact of Bisphenol A, an endocrine disruptor (BPA) present in thermal papers, on individual human red blood cells has been investigated using Raman Tweezers [29]. Significant intensity variations were evident for hemoglobin oxygenation markers in the Raman spectra of RBCs in bisphenol treated cells. RBC hemoglobin depletion was also noted in the presence of BPA. RBCs have undergone morphological changes in response to BPA in vitro exposures, which finally lead to cell bursting at higher doses. These studies have altogether proved the potential application of Raman Tweezers system for studying live cells and their interactions. The present study is an extension of above reported works in view of its relevance to transfusion medicine. Deoxygenation of hemoglobin in red blood cells is also observed here, in case of the clinically used dextrose based IV fluids with respect to blood plasma. Heme aggregation was also observed for RBCs suspended in DNS, which is probed by Raman Tweezers study.

\section{Conclusions}

The present study is an investigation on the behavior of red blood cells in dextrose containing intravenous fluids and blood plasma in an optical trap probed by microRaman spectroscopy. Hemoglobin deoxygenation trend is observed for red blood cells in presence of dextrose containing intravenous fluids. The present study indicates that blood plasma is able to maintain the oxygenation state even in an optical trap. At the same time, RBCs suspended in dextrose containing IV fluids undergo deoxygenation most readily. This also hints the probability of weak oxygen binding affinity of red blood cells in the intravenous fluids. Variations in Raman signatures (markers) were sufficient enough to provide significant discrimination amongst different groups using Principle Component Analysis. Distortion in the normal discocyte shape of RBC was evident in phase 
imaging measurements in both dextrose containing IV fluids. This study underpins the necessity of conducting systematic investigations to evaluate the influence of intravenous fluids on red blood cells. However, further biological studies are needed to elucidate the detailed mechanisms.

\section{Disclosures}

All authors declare that there is no conflict of interests in this paper.

\section{Acknowledgments}

Authors are thankful to DBT, DST-FIST, Govt. of. India and VGST, Govt. of Karnataka for providing the financial support for the fellows working in the project. Jijo Lukose also acknowledge Manipal Academy of Higher Education for the Post- Doctoral fellowship. Authors also like to acknowledge Vittal Shenoy and the staffs in Blood Bank, Kasturba Medical College, Manipal for their support during this research work.

\section{References}

1. R. Smith, K. L. Wright, and L. Ashton, "Raman Spectroscopy: An evolving technique for live cell studies,” Analyst 141(12), 3590-3600 (2016).

2. P. Strobbia, R. Odion, and T. Vo-Dinh, "Spectroscopic Chemical Sensing and Imaging: From Plants to Animals and Humans," Chemosensors 6(1), 11 (2018).

3. H. J. Butler, L. Ashton, B. Bird, G. Cinque, K. Curtis, J. Dorney, K. Esmonde-White, N. J. Fullwood, B. Gardner, P. L. Martin-Hirsch, M. J. Walsh, M. R. McAinsh, N. Stone, and F. L. Martin, "Using Raman spectroscopy to characterize biological materials," Nature protocols 11(4), 664-687 (2016).

4. Y. Kumamoto, Y. Harada, T. Takamatsu, and H. Tanaka, "Label-free Molecular Imaging and Analysis by Raman Spectroscopy," Acta histochemica et cytochemica 51(3), 101-110 (2018).

5. E. Brauchle, K. Schenke-Layland, "Raman spectroscopy in biomedicine - non-invasive in vitro analysis of cells and extracellular matrix components in tissues," Biotechnology journal 8(3), 288-297 (2013).

6. A. Ashkin, J. M. Dziedzic, J. E. Bjorkholm, and S. Chu, "Observation of a single-beam gradient force optical trap for dielectric particles," Optics letters 11(5), 288 (1986).

7. A. Ashkin, J. M. Dziedzic, "Optical trapping and manipulation of viruses and bacteria," Science 235(4795), 1517 (1987).

8. A. Ashkin, J. M Dziedzic, and T. Yamane, "Optical trapping and manipulation of single cells using infrared laser beams," Nature 330(6150), 769 (1987).

9. C. Xie, C. Goodman, M. A. Dinno, and Y.-Q. Li, "Real-time Raman spectroscopy of optically trapped living cells and organelles," Optics Express 12 (25), 6208 (2004).

10. C. Xie, D. Chen, and Y.-q. Li, "Raman sorting and identification of single living micro-organisms with optical tweezers," Optics Letters 30(14), 1800 (2005).

11. Y. M, K. Chawla, A. Bankapur, M. Acharya, J. S. D’Souza, and S. Chidangil, "A micro-Raman and chemometric study of urinary tract infection-causing bacterial pathogens in mixed cultures," Analytical and Bioanalytical Chemistry 411(14), 3165-3177 (2019).

12. R. Liu, Z. Mao, D. L. Matthews, C.-S. Li, J. W. Chan, and N. Satake, "Novel single-cell functional analysis of red blood cells using laser tweezers Raman spectroscopy: Application for sickle cell disease," Experimental hematology 41(7), 656-661.e1 (2013).

13. A. C. De Luca, G. Rusciano, R. Ciancia, V. Martinelli, G. Pesce, B. Rotoli, L. Selvaggi, and A. Sasso, "Spectroscopical and mechanical characterization of normal and thalassemic red blood cells by Raman Tweezers," Optics Express 16(11), 7943 (2008).

14. S. Barkur, D. Mathur, and S. Chidangil, "A laser Raman tweezers study of eryptosis," Journal of Raman Spectroscopy 49(7), 1155-1164 (2018).

15. A. Bankapur, S. Barkur, S. Chidangil, and D. Mathur, "A micro-raman study of live, single Red Blood Cells (RBCs) treated with AgNO3 nanoparticles," PLoS One 9(7), e103493 (2014).

16. G. Rusciano, A. C. De Luca, G. Pesce, and A. Sasso, "Raman Tweezers as a Diagnostic Tool of HemoglobinRelated Blood Disorders," Sensors 8, 7818 (2008)

17. R. Dasgupta, R. S. Verma, S. Ahlawat, A. Uppal, and P. K. Gupta, "Studies on erythrocytes in malaria infected blood sample with Raman optical tweezers," Journal of Biomedical Optics 16(7), 077009 (2011).

18. S. Rao, Š. Bálint, B. Cossins, V. Guallar, and D. Petrov, "Raman Study of Mechanically Induced Oxygenation State Transition of Red Blood Cells Using Optical Tweezers,” Biophysical Journal 96(1), 209-216 (2009).

19. D. Lin, Z. Zheng, Q. Wang, H. Huang, Z. Huang, Y. Yu, S. Qiu, C. Wen, M. Cheng, and S. Feng, "Label-free optical sensor based on red blood cells laser tweezers Raman spectroscopy analysis for ABO blood typing," Optics Express 24 (21), 24750 (2016). 
20. J. Lin, L. Shao, S. Qiu, X. Huang, M. Liu, A. Zheng, D. Lin, Y. Xu, Z. Li,Y. Lin, R. Chen, and S. Feng, "Application of a near-infrared laser tweezers Raman spectroscopy system for label-free analysis and differentiation of diabetic red blood cells," Biomedical Optics Express 9(3), 984 (2018).

21. Intravenous Fluid Therapy: Intravenous Fluid Therapy in Adults in Hospital [Internet], NICE Clinical Guidelines № 174, London, Royal College of Physicians (UK), 2013.

22. S. Pandya, Practical guidelines on fluid therapy, 2nd edition, Bhalani (2005).

23. K. J. Chin, J. Macachor, K. C. Ong, and B. C. Ong, "A comparison of $5 \%$ dextrose in $0.9 \%$ normal saline versus non-dextrose-containing crystalloids as the initial intravenous replacement fluid in elective surgery," Anaesthesia and intensive care 34(5), 613 (2006).

24. B. R. Wood, D. McNaughton, "Micro-Raman characterization of high- and low- spin heme moieties within single living erythrocytes," Biopolymers 67(4-5), 259-262 (2002).

25. B. R. Wood, B. Tait, and D. McNaughton, "Micro-Raman characterisation of the R to $\mathrm{T}$ state transition of haemoglobin within a single living erythrocyte," Biochimica et Biophysica Acta (BBA)-Molecular Cell Research $1539(1-2), 58(2001)$.

26. C. G. Atkins, H. G. Schulze, D. Chen, D. V. Devine, M. W. Blades, and R. F. B. Turner, "Using Raman spectroscopy to assess hemoglobin oxygenation in red blood cell concentrate: an objective proxy for morphological index to gauge the quality of stored blood ?" Analyst 142(12), 2199-2210 (2017).

27. B. R. Wood, P. Caspers, G. J. Puppels, S. Pandiancherri, and D. McNaughton, "Resonance Raman spectroscopy of red blood cells using near-infrared laser excitation," Analytical and bioanalytical chemistry 387(5), 1691 (2007).

28. J. L. Deng, Q. Wei, M. H. Zhang, Y. Z. Wang, and Y. Q. Li, "Study of the effect of alcohol on single human red blood cells using near - infrared laser tweezers Raman spectroscopy," Journal of Raman Spectroscopy 36(3), 257 (2005).

29. J. Lukose, N. Mithun, M. Priyanka, G. Mohan, S. Shastry, and S. Chidangil, "Laser Raman tweezer spectroscopy to explore the bisphenol A-induced changes in human erythrocytes," RSC Advances 9(28), 15933 (2019).

30. R. Wood, L. Hammer, L. Davis, and D. McNaughton, "Raman microspectroscopy and imaging provides insights into heme aggregation and denaturation within human erythrocytes," Journal of biomedical optics 10(1), 014005 (2005).

31. C. Uzoigwe, "The human erythrocyte has developed the biconcave disc shape to optimise the flow properties of the blood in the large vessels," Medical hypotheses 67(5), 1159 (2006).

32. S. Barkur, J. Lukose, and S. Chidangil, "Probing Nanoparticle-Cell Interaction Using Micro-Raman Spectroscopy: Silver and Gold Nanoparticle-Induced Stress Effects on Optically Trapped Live Red Blood Cells," ACS Omega 5(3), $1439(2020)$.

33. J. Lukose, M. N, G. Mohan, S. Shastry, and S. Chidangil, "Normal saline-induced deoxygenation of red blood cells probed by optical tweezers combined with the micro-Raman technique," RSC Advances 9(14), 7878 (2019).

34. J. Lukose, M. N, G. Mohan, S. Shastry, and S. Chidangil, "Optical tweezers combined with micro - Raman investigation of alcohol - induced changes on single, live red blood cells in blood plasma," Journal of Raman Spectroscopy 50(10), 1367 (2019). 